(2) Open Access Full Text Article

\title{
A retrospective analysis of the clinicopathological and molecular characteristics of pulmonary blastoma
}

\author{
Yuan-Yuan Zhao ${ }^{1, *}$ \\ Lin Liu ${ }^{1,2, *}$ \\ Ting Zhou ${ }^{1, *}$ \\ Ning-Ning Zhou' \\ Yun-Peng Yang' \\ Xue Hou' \\ Yong $\mathrm{Li}^{3}$ \\ Hong-Yun Zhao' \\ Yan Huang' \\ Li Zhang' \\ 'Department of Medical Oncology, \\ Sun Yat-Sen University Cancer \\ Center, State Key Laboratory \\ of Oncology in South China, \\ Collaborative Innovation Center \\ for Cancer Medicine, Guangzhou, \\ ${ }^{2}$ Department of Medical Oncology, \\ The Fifth Affiliated Hospital of \\ Sun Yat-Sen University, Zhuhai, \\ Guangdong, ${ }^{3}$ Department of \\ Pathology, Sun Yat-Sen University \\ Cancer Center, State Key Laboratory \\ of Oncology in South China, \\ Collaborative Innovation Center \\ for Cancer Medicine, Guangzhou, \\ People's Republic of China \\ *These authors contributed equally \\ to this work
}

Correspondence: Li Zhang Department of Medical Oncology, Sun Yat-Sen University Cancer Center, State Key Laboratory of Oncology in South China, 65I Dong Feng Road East, Guangzhou 510060, People's Republic of China

Tel +86 2087343822

Fax +86 2087343365

Email zhangli@sysucc.org.cn
This article was published in the following Dove Press journal:

OncoTargets and Therapy

8 November 2016

Number of times this article has been viewed

Purpose: The aim of this study was to analyze and summarize the clinicopathological and molecular characteristics of classic biphasic pulmonary blastoma (PB) to improve its diagnosis and treatment.

Patients and methods: A retrospective analysis was performed in patients who were diagnosed with PB at Sun Yat-Sen University Cancer Center from March 1995 to March 2015. Genomic DNA was profiled using a capture-based targeted sequencing panel.

Results: Sixteen patients with an average age of 40 years were included in this study. Accurate preoperative diagnosis was very challenging as surgically resected tissues with immunohistochemical staining were required for the diagnosis. Surgery was the optimal treatment for localized disease and there was no standard management for metastatic disease. Mutations were detected among 9 out of the 56 genes profiled, including BRCA2, ERBB4, ALK, MET, BRAF, RAF1, PTEN, EGFR, and PIK3CA.

Conclusion: Due to the low incidence rate and the reclassification of $\mathrm{PB}$, no standard treatment is available. Although the numbers of cases are few with varying individual experiences, it is important to improve our understanding regarding this rare lung cancer. Targeted DNA sequencing may be of clinical use for molecular testing and the effects of targeted therapy need to be confirmed.

Keywords: pulmonary blastoma, targeted DNA sequencing, clinicopathological characteristics, molecular characteristics

\section{Introduction}

Pulmonary blastoma $(\mathrm{PB})$ is a very rare pulmonary neoplasm, similar to fetal lung tissues, comprising $0.25 \%-0.5 \%$ of all primary lung tumors. ${ }^{1}$ In 1952 , Barnard ${ }^{2}$ reported this disease for the first time and called it "embryoma of lung". Since its identification, PB has been classified into three subgroups: 1) classic biphasic PB, 2) well-differentiated fetal adenocarcinoma (WDFA), which is also called monophasic PB, and 3) pleuropulmonary blastoma (PPB), which generally occurs during childhood. ${ }^{3}$

However, according to the 1999 World Health Organization (WHO) classification of lung tumors, WDFAs and PPB are no longer regarded as biphasic tumors. ${ }^{4}$ WDFA is included as a histological variant of adenocarcinoma and should not be regarded as a "monophasic" PB. PPB is classified as soft tissue tumors, occurring in children younger than 6 years of age.

Currently, PB is regarded as a type of sarcomatoid carcinomas. It is a biphasic tumor containing fetal adenocarcinoma (typically of low grade) and primitive mesenchymal stroma., 
Due to its rarity, exploring the clinical features of this tumor is challenging and only few studies have focused on gene alterations of PB. In this study, we retrospectively reviewed 16 cases with $\mathrm{PB}$ and reported for the first time the gene profile of PB based on targeted DNA sequencing.

\section{Patients and methods}

\section{Patients}

From March 1995 to March 2015, 25 patients were diagnosed with PB after histopathological examination in Sun Yat-Sen University Cancer Center. Of these 25 patients, 8 had PPB and 1 had WDFA. Therefore, these patients were excluded from this study according to WHO classification. ${ }^{5}$ The remaining 16 patients diagnosed with $\mathrm{PB}$ were retrospectively analyzed. The protocol was approved by the Ethical Committee of the Cancer Center of Sun Yat-Sen University and written informed consent was obtained from each patient.

\section{Tissue samples and DNA extraction}

Formalin-fixed and paraffin-embedded tissue sections were obtained from 5 of the 16 patients. The five patients were numbered according to the time of diagnosis. DNA was extracted using a QIAamp DNA Mini Kit (Qiagen, Heidelberg, Germany) following the manufacturer's protocol.

\section{Targeted DNA sequencing}

Genomic DNA was profiled by using a capture-based targeted sequencing panel (Burning Rock Biotech, Guangzhou, People's Republic of China). A total of 56 cancer-associated genes were analyzed, of which 7 were associated with non-small-cell lung cancer (NSCLC) according to the National Comprehensive Cancer Network guidelines - EGFR, ALK, BRAF, ERBB2, MET, RET, and ROS1. Related oncogene and cancer suppressor genes were also selected in the panel (Table 1).

Qubit dsDNA assay (Invitrogen TM; Thermo Fisher Scientific, Carlsbad, CA, USA) was used to measure the concentration of the DNA samples to make sure that genomic
DNA was greater than $40 \mathrm{ng}$. Fragments of 200- to 400-bp sizes were selected with beads (Agencourt AMPure XP kit; Beckman-Coulter, Brea, CA, USA), followed by hybridization with the capture probes baits, hybrid selection with magnetic beads, and polymerase chain reaction (PCR) amplification. The quality and size range were assessed using a bioanalyzer high-sensitivity DNA assay. The available indexed samples were then loaded onto the flow cells for sequencing on a Next seq (Illumina, San Diego, CA, USA) with pair-end reads. ${ }^{6,7}$

\section{Sequence data analysis}

Sequence data were mapped to the human genome (hg19) with the BWA (Burrows-Wheeler Aligner) aligner 0.7.10. Local alignment optimization, variant calling, and annotation were performed with GATK 3.2. DNA translocation analysis was performed by using both Tophat 2 and Factera 1.4.3. ${ }^{7,8}$

\section{Results}

\section{Clinical characteristics}

Of the selected 16 patients, 10 were male and 6 were female. Their age ranged between 29 and 58 years, with an average age of 40 years (Table 2). The most common symptoms present in every patient were cough and hemoptysis. Chest pain and dyspnea were found in one case. One of these patients suffered from pneumothorax.

\section{Pathological diagnosis}

Even with the development of modern diagnostic imaging and biopsy techniques, preoperative diagnosis is still challenging because small biopsy specimens do not contain enough epithelial and mesenchymal tumor tissues. Five of the patients were diagnosed by electronic bronchoscopy and 2 by needle biopsy before the surgery. All the patients were misdiagnosed for PB at first except for one.

Pathology of the surgically resected tissue stained with hematoxylin and eosin (H\&E) demonstrated a typical biphasic tumor - one was mesenchymal malignancies that

Table I Genes panel for targeted DNA sequencing

\begin{tabular}{|c|c|c|c|}
\hline Gene associated with NSCLC & Oncogene & Cancer suppressor gene & $\begin{array}{l}\text { Gene associated with } \\
\text { drug metabolism } \\
\text { (SNP) }\end{array}$ \\
\hline $\begin{array}{l}\text { EGFR, ALK, BRAF, ERBB2, MET, } \\
\text { RET, ROSI }\end{array}$ & $\begin{array}{l}\text { AKTI, ARAF, CCNDI, CDK4, CDK6, CTNNBI, } \\
\text { DDR2, ERBB3, ERBB4, FGF3, FGF4, FGFI9, } \\
\text { FGFRI, FGFR2, FGFR3, FLT3, HRAS, JAKI } \\
\text { JAK2, KDR, KIT } \\
\text { KRAS, MAP2KI, MTOR, MYC, NRAS, NRGI, } \\
\text { NTRKI, NTRK2, NTRK3, PDGFRA, PIK3CA, } \\
\text { PTCHI, RAFI, SMO }\end{array}$ & $\begin{array}{l}\text { ATM, BIM, BRCAI, BRCA2, } \\
\text { CDKN2A, PTEN, RBI, } \\
\text { STKII, TP53, TSCI, TSC2 }\end{array}$ & CYP2D6, DPYD, UTGIAI \\
\hline
\end{tabular}

Abbreviations: SNP, single nucleotide polymorphism; NSCLC, non-small-cell lung carcinoma. 
Table 2 Characteristics of 16 patients when diagnosed with pulmonary blastoma

\begin{tabular}{lll}
\hline Characteristics & Subset & Number of patients \\
\hline Median (range) age in years & & $40(29-58)$ \\
Sex & Male & 10 \\
& Female & 6 \\
Smoking status & Nonsmokers and & 8 \\
& mild smokers & \\
Smokers & 8 \\
ECOG PS & $0-$ I & 15 \\
Disease stage & 2 & 1 \\
& I-III & 9 \\
& IV & 5 \\
\hline
\end{tabular}

Abbreviation: ECOG PS, Eastern Cooperative Oncology Group performance status. consist of round or shuttle cells with atypia nuclear and visible pathological karyokinesis and the other was epithelial tumors composed of infrequent tubular and glandular structures. Immunohistochemical staining of the tumor specimen showed that epithelial markers such as CK, EMA, and TTF-1 were positive for the epithelial component whereas vimentin was positive for mesenchymal areas (Figure 1A-F).

\section{Image examination and tumor marker}

Radiological examinations (enhanced computed tomography or positron emission tomography [CT/PET]) revealed pulmonary mass ranging from 35 to $110 \mathrm{~mm}$, and the
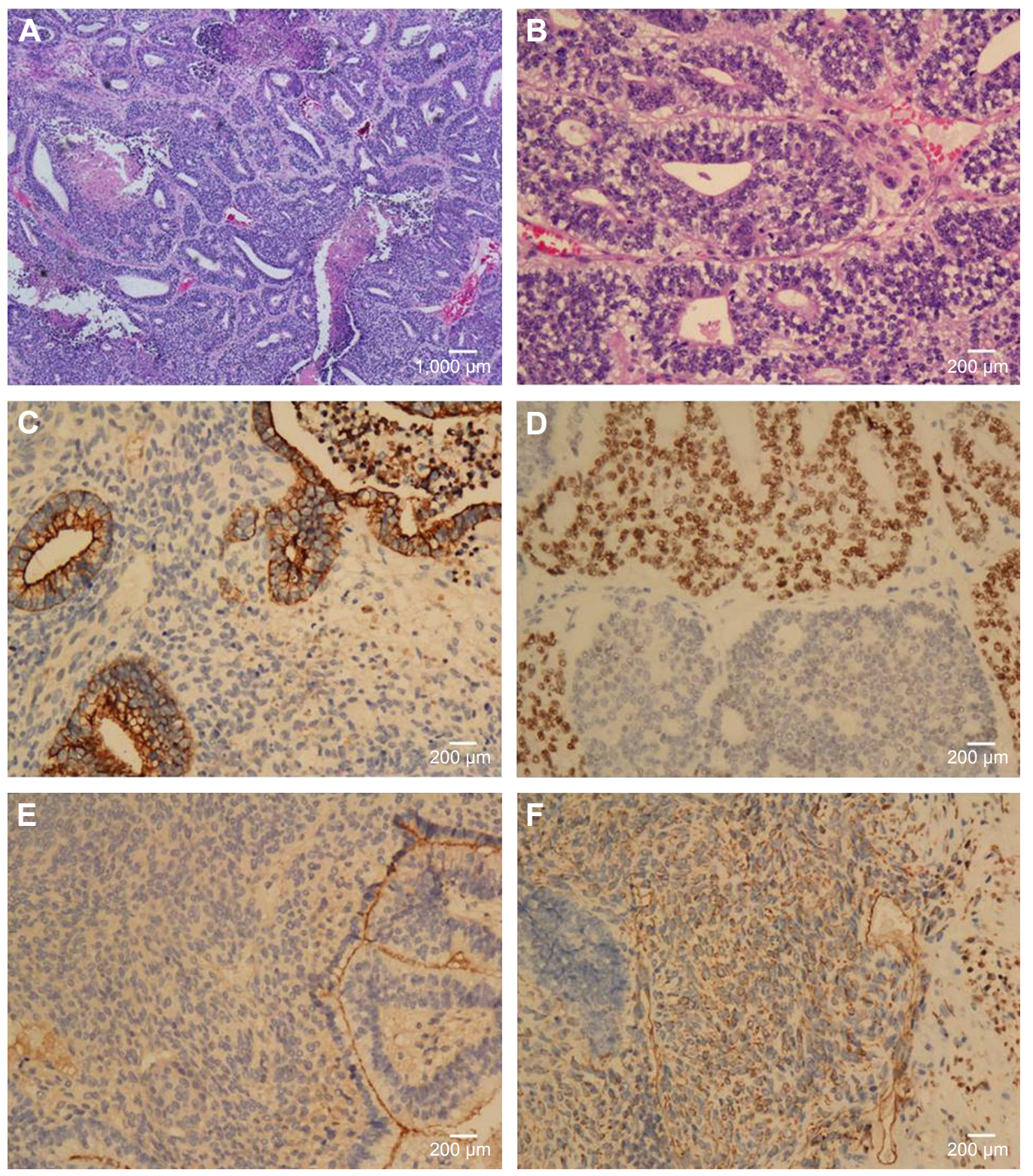

Figure I Pathological examination (hematoxylin and eosin staining) shows a part of carcinoma with slit-formed duct formation and immature mesenchymal cells. (A) 40 $\times$ and (B) 200x. The malignant glandular component was diffusely positive for epithelial marker (200x): (C) CK, (D) TTF-I, (E) EMA. The malignant stromal component was positive for mesenchymal marker (200x), (F) Vimentin. 

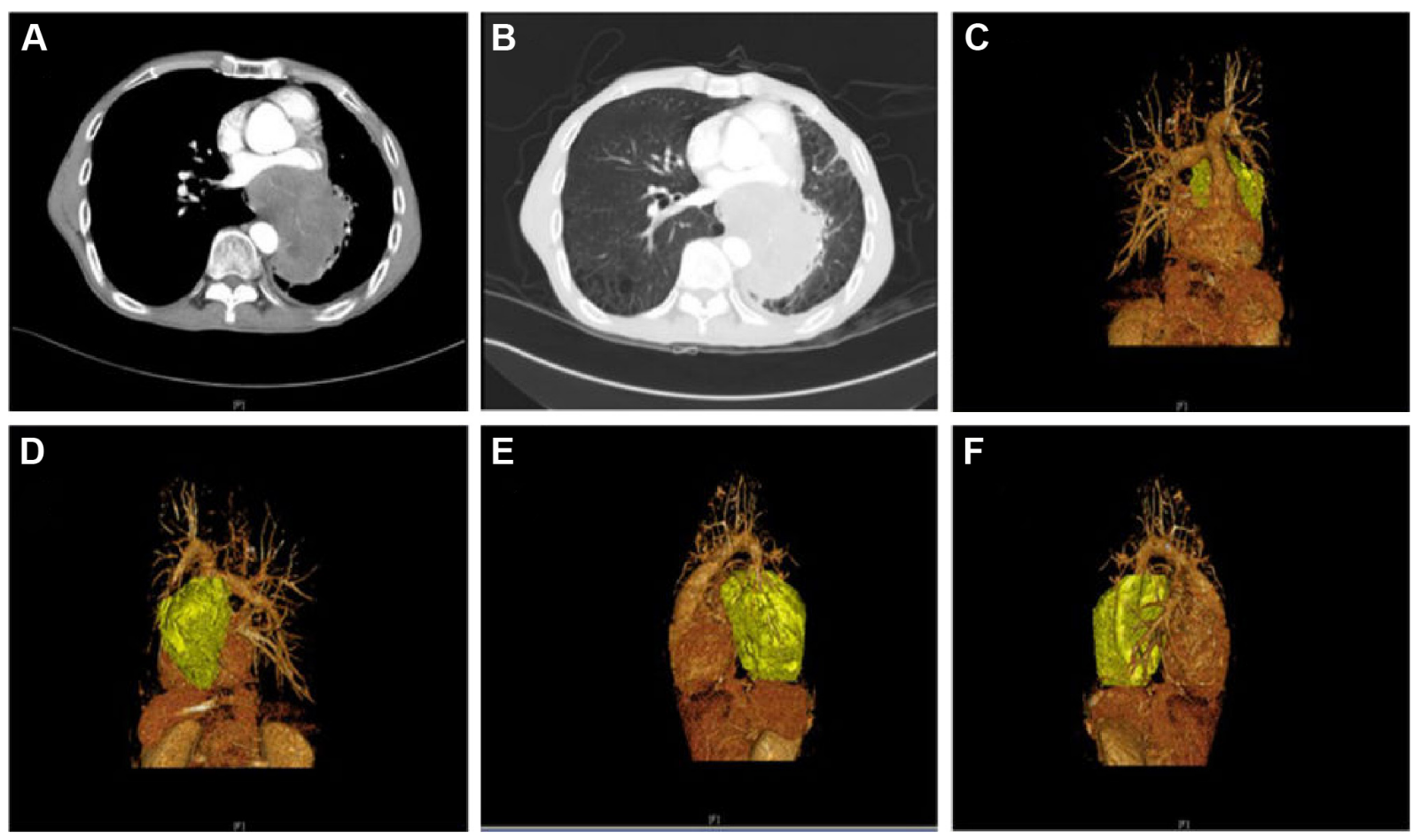

Figure 2 Typical CT (computed tomography) images of one of our patients. The CT scan revealed a mass opacity of diameter $80 \times 70 \mathrm{~mm}$ in size in the lower lobe of the left lung near the hilar area. (A) Mediastinal window, (B) lung window, and (C-F) CT 3-dimensional reconstructions.

medium diameter was $55 \mathrm{~mm}$. Typical CT images of one of the patients are shown in Figure 2. At the initial diagnosis, mediastinum lymph nodes were found in 3 patients; bone, bilateral adrenal gland metastasis, and intrapulmonary metastasis were found in one patient. Several serum tumor markers including AFP, NSE, CEA, CA199, CA153, CA125, and $\beta$-HCG were tested. Increasing CA199 and NSE were observed but had no specific significance.

\section{Treatment}

Most of the patients (14/16) underwent surgery. The combination drugs that were used in adjuvant or first-line chemotherapy are cyclophosphamide, doxorubicin, and cisplatin; cyclophosphamide, doxorubicin, vincristine/ ifosfamide, etoposide alternation, etoposide, ifosfamide, cisplatin, paclitaxel and cisplatin, and gemcitabine and cisplatin. Paclitaxel, gemzar, and docetaxel were also used in second- or third-line chemotherapy. One patient received gefitinib for 9 months as fourth-line treatment. No patients received thoracic radiotherapy after the surgery. Whole brain radiotherapy was conducted in 3 patients with brain metastasis.

\section{Distance metastasis and overall survival}

Progression was detected through regular screening during or after treatment. According to the investigation, sites of metastasis included brain, bone, adrenal gland, and liver. Overall survival (OS) was measured from the time of diagnosis to death. The median follow-up duration was 26 months. The median OS was 36 months.

\section{Results of targeted DNA sequencing}

A gene mutation profiling was performed by targeted DNA sequencing. Mutations were detected for 9 out of the 56 genes profiled-BRCA2, ERBB4, ALK, MET, BRAF, RAF1, PTEN, EGFR, and PIK3CA (Table 3). Four gene alterations with high frequency were found in only 1 patient. The tumor of patient (patient number 2) who received gefitinib for 9 months as fourth-line treatment had 3 different $A L K$ gene mutations but no EGFR mutation.

\section{Discussion}

Pulmonary sarcomatoid carcinoma (PSC) belongs to highly aggressive and poorly differentiated NSCLC and PB is one of its subtypes. ${ }^{9}$ Due to the low incidence rate and reclassification of the disease, it is difficult to interpret the epidemiology, clinical features, treatment, and prognosis of PB. A lot of earlier reports of PB have included fetal adenocarcinomas, so data are not in accordance to the new classification. ${ }^{10}$ Considering all these limits, even small number of cases or individual experiences of $\mathrm{PB}$ 
Table 3 Results of targeted DNA sequencing of 5 patients with available DNA

\begin{tabular}{|c|c|c|c|c|c|}
\hline $\begin{array}{l}\text { Patient } \\
\text { number }\end{array}$ & Gender & $\begin{array}{l}\text { Age } \\
\text { (years) }\end{array}$ & Gene & Mutation type & Frequency (\%) \\
\hline \multirow[t]{4}{*}{ I } & Male & 40 & BRCA2 & Exon 27 & 50.2 \\
\hline & & & & D34I0G missense mutation & \\
\hline & & & ERBB4 & Exon 12 & 3.25 \\
\hline & & & & R488W missense mutation & \\
\hline \multirow[t]{4}{*}{2} & Female & $4 \mid$ & $A L K$ & Exon 23 & 5.95 \\
\hline & & & & VIII80L missense mutation & \\
\hline & & & & LOCI 02723526-ALK fusion & 6.06 \\
\hline & & & & MAST4-ALK fusion & 7.87 \\
\hline \multirow[t]{8}{*}{3} & Female & 29 & MET & Exon 15 & 73.5 \\
\hline & & & & PI045S missense mutation & \\
\hline & & & BRAF & Exon 15 & 63.0 \\
\hline & & & & V600E missense mutation & \\
\hline & & & RAFI & Exon 13 & 60.5 \\
\hline & & & & L465R missense mutation & \\
\hline & & & PTEN & Exon 5 & 26.7 \\
\hline & & & & GVMII 32 frameshift mutation & \\
\hline \multirow[t]{4}{*}{4} & Female & 34 & EGFR & Exon 20 & 28.5 \\
\hline & & & & M766MASV noncode insertion mutation & \\
\hline & & & PIK3CA & Exon 10 & 5.73 \\
\hline & & & & E545A missense mutation & \\
\hline 5 & Male & 37 & None & & \\
\hline
\end{tabular}

are important to improve our understanding of this rare lung cancer.

The average age was 39 years for the occurrence of classic biphasic PB as reported by Van Loo et al. ${ }^{11}$ The recurrence rate was $43 \%$, and the tumor showed a tendency to metastasize during recurrence in the brain, mediastinum, pleura, liver, diaphragm, heart, and soft tissues of the extremities. ${ }^{12}$ The average age of patients included in this study was 40 years, which is consistent with the earlier studies. Most of the patients finally developed distant metastasis.

Surgery is the optimal treatment for localized disease, ${ }^{13}$ and there is no standard management for metastatic disease. Patients may receive combination of surgery and chemotherapy and radiotherapy, just as patients in this study. Currently, markable advances have been made in the development of targeted agents for the treatment of molecularly defined subsets of NSCLC. However, the effect of targeted therapy in PB is unclear with very few case reports found in the literature. Carboplatin and paclitaxel plus bevacizumab were reported to be effective in $1 \mathrm{~PB}$ patient with distant metastasis, but finally failed after the fourth course. ${ }^{14}$ Sorafenib was also reported to be useful in 1 patient with biphasic PB with renal metastasis. ${ }^{15}$ MET mutations leading to exon 14 skipping were reported to be frequent $(22 \%)$ and potentially targetable events in PSC. ${ }^{9}$ One patient diagnosed with PSC with
MET amplification and mutation responded to crizotinib. However, carcinosarcoma and blastoma were excluded in this study. ${ }^{9}$ Further studies are required to confirm the effects of targeted therapy on PB.

Targeted DNA sequencing provides a potentially important diagnostic test that allows the recognition of the abnormalities in each patient. Such multiplex genomic testing will assist physicians to identify actionable mutations with available targeted treatments or new target agents for clinical trials. ${ }^{16}$ One limitation of this study was its retrospective nature. Otherwise, patient (patient number 3) with BRAF V600E mutation may benefit from BRAF inhibitors, ${ }^{17}$ while patient (patient number 2) with ALK fusion tumors may try crizotinib $^{18}$ or ceritinib ${ }^{19}$ first if available when there were fewer standard therapeutic options left. The other limitation of this study was its small sample size due to the low incidence rate of the disease. Although with limitations mentioned above, this is the first study to provide the molecular profile of PB using targeted DNA sequencing. The results, however, have to be verified by further research.

\section{Conclusion}

In summary, the occurrence of $\mathrm{PB}$ is rare and there is no standard treatment available for this metastatic disease. With the challenging nature of its histology, it is difficult to diagnose this disease with a small biopsy specimen. This study suggests that targeted DNA sequencing may be of clinical 
use for molecular testing. However, the effects of targeted therapy need to be confirmed by further research.

\section{Acknowledgments}

The authors thank Burning Rock Biotech, Guangzhou, People's Republic of China, for their cooperation in targeted DNA sequencing and also they thank Dr Ying Chen and Dr Jia-xing Huang for their hard work in the data collection process. This work was funded by the Natural Science Foundation of Guangdong Province (2014A030310030) and the China National Natural Science Foundation (81502352 and 81501986).

\section{Disclosure}

The authors report no conflicts of interest in this work.

\section{References}

1. Francis D, Jacobsen M. Pulmonary blastoma. Cur Top Pathol. 1983; 73:265-294.

2. Barnard WG. Embryoma of lungs. Thorax. 1952;7(4):299-301.

3. Koss MN, Hochholzer L, O'Leary T. Pulmonary blastomas. Cancer. 1991; 67(9):2368-2381.

4. Brambilla E, Travis WD, Colby TV, Corrin B, Shimosato Y. The new World Health Organization classification of lung tumours. Eur Respir J. 2001;18(6):1059-1068.

5. Travis WD, Brambilla E, Nicholson AG, et al. The 2015 World Health Organization classification of lung tumors: impact of genetic, clinical and radiologic advances since the 2004 classification. J Thorac Oncol. 2015;10(9):1243-1260.

6. Yu Y, Dong L, Li D, et al. Targeted DNA sequencing detects mutations related to susceptibility among familial non-medullary thyroid cancer. Sci Rep. 2015;5:16129.
7. Cai $\mathrm{W}, \mathrm{Lin} \mathrm{D}, \mathrm{Wu} \mathrm{C}$, et al. Intratumoral heterogeneity of ALK-Rearranged and ALK/EGFR coaltered lung adenocarcinoma. J Clin Oncol. 2015; 33(32):3701-3709.

8. Frampton GM, Fichtenholtz A, Otto GA, et al. Development and validation of a clinical cancer genomic profiling test based on massively parallel DNA sequencing. Nat Biotech. 2013;31(11):1023-1031.

9. Liu X, Jia Y, Stoopler MB, et al. Next-generation sequencing of pulmonary sarcomatoid carcinoma reveals high frequency of actionable MET gene mutations. J Clin Oncol. 2016;34(8):794-802.

10. Smyth RJ, Fabre A, Dodd JD, Bartosik W, Gallagher CG, McKone EF. Pulmonary blastoma: a case report and review of the literature. $B M C$ Res Notes. 2014;7:294.

11. Van Loo S, Boeykens E, Stappaerts I, Rutsaert R. Classic biphasic pulmonary blastoma: a case report and review of the literature. Lung Cancer. 2011;73(2):127-132.

12. Novotny JE, Huiras CM. Resection and adjuvant chemotherapy of pulmonary blastoma: a case report. Cancer. 1995;76(9):1537-1539.

13. Xiu Y, Jiang L, Liu W. Classic biphasic pulmonary blastoma with brain and axillary metastases: a case report with molecular analysis and review of literature. Int J Clin Exp Pathol. 2015;8(1):983-988.

14. Sakata S, Saeki S, Hirooka S, Hirosako S, Ichiyasu H, Kohrogi H. A case of biphasic pulmonary blastoma treated with carboplatin and paclitaxel plus bevacizumab. Case Rep Oncol Med. 2015;2015:842621.

15. Mulamalla K, Truskinovsky AM, Dudek AZ. Pulmonary blastoma with renal metastasis responds to sorafenib. J Thorac Oncol. 2007;2(4): 344-347.

16. Takeda M, Sakai K, Terashima M, et al. Clinical application of amplicon-based next-generation sequencing to therapeutic decision making in lung cancer. Ann Oncol. 2015;26(12):2477-2482.

17. Falchook GS, Long GV, Kurzrock R, et al. Dabrafenib in patients with melanoma, untreated brain metastases, and other solid tumours: a phase 1 dose-escalation trial. Lancet. 2012;379(9829):1893-1901.

18. Kwak EL, Bang YJ, Camidge DR, et al. Anaplastic lymphoma kinase inhibition in non-small-cell lung cancer. N Engl J Med. 2010;363(18): 1693-1703.

19. Shaw AT, Kim DW, Mehra R, et al. Ceritinib in ALK-rearranged nonsmall-cell lung cancer. N Engl J Med. 2014;370(13):1189-1197.
OncoTargets and Therapy

\section{Publish your work in this journal}

OncoTargets and Therapy is an international, peer-reviewed, open access journal focusing on the pathological basis of all cancers, potential targets for therapy and treatment protocols employed to improve the management of cancer patients. The journal also focuses on the impact of management programs and new therapeutic agents and protocols on

\section{Dovepress}

patient perspectives such as quality of life, adherence and satisfaction. The manuscript management system is completely online and includes a very quick and fair peer-review system, which is all easy to use. Visit $\mathrm{http}: / /$ www.dovepress.com/testimonials.php to read real quotes from published authors. 